\title{
QCD analysis of near-threshold quarkonium leptoproduction at large photon virtualities
}

\author{
Renaud Boussarie and Yoshitaka Hatta \\ Physics Department, Brookhaven National Laboratory, Upton, New York 11973, USA
}

(Received 3 May 2020; accepted 25 May 2020; published 4 June 2020)

\begin{abstract}
We propose a novel approach to compute the cross section of near-threshold $J / \psi$ and $\Upsilon$ production in electron-proton scattering at large photon virtualities $Q^{2}$ based on an operator product expansion. We show that the process can be used to extract the gluon part of the D-term gravitational form factor of the proton. At the subleading level, it is also sensitive to the trace anomaly effect of QCD.
\end{abstract}

DOI: 10.1103/PhysRevD.101.114004

\section{INTRODUCTION}

The exclusive photoproduction of $J / \psi$ near threshold has a long history [1-3], starting almost immediately after the discovery of $J / \psi$. In the early days, it was one of the key measurements for reconfirming the existence of $J / \psi$, as well as studying its basic properties such as the coupling to hadronic matter. Over the past two decades, theoretical interest in this reaction resurfaced every once in a while [4-12] with different focuses, but it was not until recently that the subject draw a lot of attention from the viewpoint of the nucleon structure. It has been suggested theoretically $[4,9]$ that the detailed behavior of the cross section near threshold is sensitive to the trace anomaly of QCD, hence it can shed light on the origin of the proton mass (see the related works in [13-15]). This is one of the main motivations for the ongoing experiments at Jefferson laboratory (JLab) [16,17]. Moreover, the National Academy of Science in the U.S. [18] has recently identified the proton mass problem as one of the major scientific goals of the future electron-ion collider (EIC) $[19,20]$. The subject is also actively discussed in the context of the EIC in China [21]. It is then perfectly possible that the physics of near-threshold production grows into an important subfield in the EIC era.

The existing theoretical approaches are roughly divided into two categories. The one that has been used since the 1970s $[3,4,22,23]$ is to assume vector meson dominance (VMD) for the incoming photon. In this approach, the original problem $\gamma p \rightarrow J / \psi p^{\prime}$ is reduced to forward scattering $p J / \psi \rightarrow p J / \psi$ which is more amenable to various theoretical tools. A heavy quarkonium interacts with a hadron only via gluon exchanges. In the heavy quark

Published by the American Physical Society under the terms of the Creative Commons Attribution 4.0 International license. Further distribution of this work must maintain attribution to the author(s) and the published article's title, journal citation, and DOI. Funded by SCOAP. mass limit, the interaction effectively becomes local and the scattering amplitude is described by the moments of the gluon distribution function. At the subleading level, it also depends on the gluon condensate in the proton $\left\langle p\left|F^{2}\right| p\right\rangle$ $[4,24]$ which constitutes the major part of the QCD trace anomaly.

The second approach makes use of 'two-gluon form factors' $\left\langle p^{\prime}|F F| p\right\rangle[5,6,9]$. One of the distinctive features of near-threshold quarkonium production is that the momentum transfer $t=\left(p^{\prime}-p\right)^{2}$ is large. However, in the VMD approach, nonforwardness is trivialized even though the threshold value $\sqrt{-t_{\text {th }}} \approx 1.5 \mathrm{GeV}$ is comparable to the charm quark mass which is treated as the only hard scale of the problem. In [9], it has been shown via a holographic method that the amplitude is proportional to the gravitational form factor $\left\langle p^{\prime}\left|T_{\alpha \beta}^{g}\right| p\right\rangle$ where $T_{\alpha \beta}^{g}$ is the gluon part of the energy momentum tensor. Subsequently, the precise relation between the trace of $T_{\alpha \beta}^{g}$ and the gluon condensate operator $F^{2}$ has been understood [25,26]. On the other hand, $\left\langle p^{\prime}\left|T_{\alpha \beta}^{g}\right| p\right\rangle$ also contains the so-called D-term which appears only in nonforward kinematics and which has attracted a considerable attention lately (see a recent review [27] and references therein). The results of $[9,13,14]$ suggest that near-threshold quarkonium production is a unique process that can directly access not only the gluon condensate, but also the gluon D-term. The latter aspect is quite complementary to the ongoing effort to extract the quark D-term from deeply virtual Compton scattering (DVCS) [28-30]. Yet, holographic approaches are at best a model of QCD, and it remains to be seen to what extent the obtained predictions are borne out in real QCD.

Overall, the current theoretical status just described is not totally satisfactory. What is missing is a first-principle approach in QCD which can be systematically improved and compared to the data. The present work is a step toward this aim. Instead of photoproduction, we propose to study leptoproduction with large photon virtualities $q^{2}=-Q^{2}$. 
So far, leptoproduction has received remarkably little attention in the context of threshold production, perhaps because as $Q^{2}$ gets larger, one is further away from the forward kinematics. However, the large- $Q^{2}$ region appears to be the cleanest setup from a perturbative QCD point of view. We in fact consider the limit $Q^{2} \gg M^{2}$ where $M$ is the quarkonium mass.

This paper is organized as follows. In the next section we briefly review the kinematics of the reaction $\gamma p \rightarrow J / \psi p^{\prime}$ near threshold. In Sec. III, we lay out our strategy to compute the scattering amplitude based on operator product expansion (OPE). In Sec. IV, we discuss the various two-gluon form factors involved. Then in Sec. V, we numerically evaluate the cross section and study the impact of the D-term as well as the gluon condensate. Section VI is devoted to conclusions.

\section{KINEMATICS}

We shall be interested in the near-threshold production of a heavy quarkonium vector meson $H$ with mass $M$ in electron-proton scattering $e p \rightarrow e^{\prime} \gamma^{*} p \rightarrow e^{\prime} H p^{\prime}$. We have in mind $H=J / \psi$ and $\Upsilon$. The center-of-mass energy $W^{2}=$ $(q+p)^{2}$ of the virtual photon-proton subsystem at the threshold is

$$
W_{\mathrm{th}}^{2}=\left(m_{N}+M\right)^{2},
$$

where $m_{N}=0.94 \mathrm{GeV}$ is the proton mass. Numerically, $W_{t h} \approx 4.04 \mathrm{GeV}$ for $J / \psi$ and $W_{t h} \approx 10.4 \mathrm{GeV}$ for $\Upsilon . q$ and $p$ are the virtual photon and proton momenta, respectively, with $Q^{2}=-q^{2}$ being the photon virtuality. Near the threshold, the Bjorken variable takes the form

$$
x_{B}=\frac{Q^{2}}{2 p \cdot q} \approx \frac{Q^{2}}{Q^{2}+M^{2}+2 m_{N} M} .
$$

Equation (2) shows that, unlike the usual situation in DIS, $Q^{2}$ and $x_{B}$ are not independent variables. $x_{B}$ approaches unity as $Q^{2}$ goes to infinity. We also see that, somewhat counterintuitively, threshold production can occur even when $Q^{2}$ is arbitrarily large, though of course the value of $Q^{2}$ is limited in actual experiments. Using the standard variables in DIS, $S_{e p}=(p+\ell)^{2}, y=p \cdot q / p \cdot \ell$ where $\ell^{\mu}$ is the incoming electron momentum, we can write

$$
W^{2}=y\left(S_{e p}-m_{N}^{2}\right)+m_{N}^{2}-Q^{2} .
$$

Small- $W$ does not necessarily imply small- $S_{e p}$ when $Q^{2}$ is large. In particular, the process can be studied at the future EIC [31].

Let $\vec{p}_{c m}$ and $\vec{k}_{c m}$ be the 3-momentum of the incoming proton and outgoing quarkonium, respectively, in the center of mass frame of the $\gamma^{*} p$ subsystem.

$$
\begin{aligned}
p_{c m}^{2} & =\frac{W^{4}-2 W^{2}\left(m_{N}^{2}-Q^{2}\right)+\left(m_{N}^{2}+Q^{2}\right)^{2}}{4 W^{2}}, \\
k_{c m}^{2} & =\frac{\left(W^{2}-\left(M+m_{N}\right)^{2}\right)\left(W^{2}-\left(M-m_{N}\right)^{2}\right)}{4 W^{2}} .
\end{aligned}
$$

The momentum transfer is

$t=\left(\sqrt{p_{c m}^{2}+m_{N}^{2}}-\sqrt{k_{c m}^{2}+m_{N}^{2}}\right)^{2}-\left(\vec{p}_{c m}+\vec{k}_{c m}\right)^{2}$.

At the threshold, $k_{c m}=0$ so that

$t_{t h}=2 m_{N}\left(m_{N}-\sqrt{p_{c m}^{2}+m_{N}^{2}}\right)=-\frac{m_{N}\left(M^{2}+Q^{2}\right)}{m_{N}+M}$.

We see that $\left|t_{\text {th }}\right|$ is minimal in photoproduction $Q^{2}=0$ and monotonously increases with increasing $Q^{2}$. In the heavyquark mass limit, $Q^{2} \gg\left|t_{\mathrm{th}}\right|$. Away from the threshold, $t$ takes a value in the range $\left|t_{\max }\right|>|t|>\left|t_{\min }\right|$ depending on the angle between $\vec{p}_{\mathrm{cm}}$ and $\vec{k}_{\mathrm{cm}}$. The differential cross section is given by

$\frac{d \sigma_{T / L}^{\gamma^{*} p}}{d t}=\frac{\alpha_{e m}}{8\left(W^{2}-m_{N}^{2}\right) W p_{c m}} \frac{1}{2} \sum_{\text {spin }}\left|\left\langle p^{\prime} k\left|\epsilon_{q}^{T / L} \cdot J_{e m}(0)\right| p\right\rangle\right|^{2}$,

where $J_{e m}^{\mu}=\sum_{f} e_{f} \bar{q}_{f} \gamma^{\mu} q_{f}$ is the electromagnetic current ( $e_{f}$ being the charge in units of $|e|$ ) and $T / L$ refers to the transversely $(T)$ or longitudinally $(L)$ polarized virtual photon. The factor $1 / 2$ is for averaging over proton helicities. The nontrivial dynamics of QCD is contained in the hadronic matrix element

$$
\begin{aligned}
& \int d^{4} y e^{-i q \cdot y}\left\langle p^{\prime} k\left|J_{e m}^{\nu}(y)\right| p\right\rangle \\
& \quad=(2 \pi)^{4} \delta\left(k+p^{\prime}-q-p\right)\left\langle p^{\prime} k\left|J_{e m}^{\nu}(0)\right| p\right\rangle .
\end{aligned}
$$

Computing (8) from first principles in QCD is a challenging task. Most of the previous theoretical works have focused on the photoproduction limit. In contrast, in this paper we shall investigate leptoproduction in the large $Q^{2} \gg M^{2}$ region.

\section{OPE AT LARGE $Q^{2}$}

In this section, we formulate our strategy to calculate the hadronic matrix element $\left\langle p^{\prime} k\left|J_{e m}\right| p\right\rangle$ near threshold at large $Q^{2} \gg M^{2}$. We have chosen to work in the high $Q^{2}$ region for reasons to become clear shortly. For definiteness, we consider $J / \psi$ production, but the case with $\Upsilon$ is completely analogous. In fact, our approach is better justified when $M \gg m_{N}$. Thus, $\Upsilon$ production is more preferred from a theoretical point of view, though of course experimentally it is more challenging. 
Let us first mention that, if the center of mass energy is sufficiently high $s=W^{2} \gg M^{2},|t|$, the process is commonly called deeply virtual meson production (DVMP). The cross section is known to factorize in perturbative QCD in terms of the generalized parton distribution (GPD) and the meson distribution amplitude (DA) [32]. Near the threshold, $s=\mathcal{O}\left(M^{2}\right)$, and $|t|$ is comparable to, or even exceeds $s$ depending on the value of $Q^{2}$, see (6). Note, however, that $s$ is small because of the cancellation $s=$ $2 p \cdot q-Q^{2}+\cdots$ and $x_{B}=Q^{2} / 2 p \cdot q$ stays close to unity. Moreover, $2 p \cdot q \sim Q^{2} \gg|t|$ at least parametrically when $M \gg m_{N}$ [see (6)]. This gives us some hope that a perturbative approach is possible, see $[33,34]$ for an approach potentially related to the following discussion.

Our basic argument is that near the threshold, the amplitude (8) is related to the following current-current correlator

$\epsilon_{\mu}^{*}(k) i \int d^{4} x d^{4} y e^{i k \cdot x-i q \cdot y}\left\langle p^{\prime}\left|\mathrm{T}\left\{\bar{c} \gamma^{\mu} c(x) J_{e m}^{\nu}(y)\right\}\right| p\right\rangle$,

where $\epsilon_{\mu}(k)$ is the $J / \psi$ polarization vector. This matrix element is similar to the one that appears in doubly virtual Compton scattering (DDVCS) $\gamma^{*}(q) p \rightarrow \gamma^{*}(k) p^{\prime}$, or timelike Compton scattering (TCS) in the special case $q^{2}=0$ (see, e.g., [35]). However, there is a crucial difference. The DDVCS amplitude is given by the correlator $\left\langle J_{e m} J_{e m}\right\rangle$, and is dominated by the light quark degrees of freedom (light quark GPDs) except in the very small- $x_{B}$ region where it is dominated by gluons. In (9), on the other hand, one of the electromagnetic currents has been replaced by the charm quark current operator (bottom quark, in the case of $\Upsilon$ production). As a result, only the charm component of the other $J_{e m}$ is relevant, and the matrix element becomes primarily sensitive to the gluonic content of the proton.

That the $J / \psi$ production amplitude is related to a DDVCS-like (photon production) amplitude is intuitively reasonable, in view of the fact that in actual experiments, a $J / \psi$ and a timelike photon with virtuality exactly at the $J / \psi$ mass are practically indistinguishable as they are probed via leptonic final states $\left(e^{+} e^{-}\right.$pairs). However, in DDVCS or TCS, the resonance region $k^{2} \approx M^{2}$ is usually avoided because the nonperturbative final state effect to produce the vector meson comes into play. As a function of $k^{2}$, (9) has a sharp resonance peak near the $J / \psi$ mass shell. Using the LSZ reduction formula, we can write

$$
\begin{gathered}
e e_{f} \epsilon_{\mu}^{*}(k) i \int d^{4} x d^{4} y e^{i k \cdot x-i q \cdot y}\left\langle p^{\prime}\left|\mathrm{T}\left\{\bar{c} \gamma^{\mu} c(x) J_{e m}^{\nu}(y)\right\}\right| p\right\rangle \\
\approx \frac{g_{\gamma J / \psi}}{k^{2}-M^{2}+i M \Gamma} \int d^{4} y e^{-i q \cdot y}\left\langle p^{\prime} k\left|J_{e m}^{\nu}(y)\right| p\right\rangle
\end{gathered}
$$

where $e_{f}=2 / 3$ for the charm quark and $\Gamma$ is the total width of $J / \psi$. The decay constant is related to the electromagnetic width as

$$
\Gamma_{e^{+} e^{-}}=\frac{\alpha_{e m} g_{\gamma J / \psi}^{2}}{3 M^{3}}
$$

Away from the very narrow peak (note that $\Gamma=93 \mathrm{keV} \ll M=3.1 \mathrm{GeV}$ ), the current correlator is expected to behave smoothly. We thus arrive at the relation

$$
\int d^{4} y e^{-i q \cdot y}\left\langle p^{\prime} k\left|J_{e m}^{\nu}(y)\right| p\right\rangle=\beta \frac{e e_{f} M^{2}}{g_{\gamma J / \psi}} \epsilon_{\mu}^{*}(k) i \int d^{4} x d^{4} y e^{i k \cdot x-i q \cdot y}\left\langle p^{\prime}\left|\mathrm{T}\left\{\bar{c} \gamma^{\mu} c(x) J_{e m}^{\nu}(y)\right\}\right| p\right\rangle,
$$

where $\beta$ is a c-number of order unity which is not under control. It is understood that the right-hand side is evaluated close to, but not too close to the $J / \psi$ mass shell $\left|k^{2}-M^{2}\right| \gg M \Gamma$. Our key observation is that in this offmass-shell region, one can perform an operator product expansion (OPE) when $Q^{2}$ is large.

Before doing so, a few additional remarks are in order. (i) On general grounds, one expects corrections to (10) from higher resonances which the operator $\bar{c} \gamma^{\mu} c$ can excite. However, this effect will be suppressed near threshold because, at fixed values of $s$ and $t$, only the resonances with mass smaller than $\sqrt{s}-m_{N}$ can be produced. There may also be contributions from the deep Euclidean region $k^{2}<0$ if one considers a dispersion relation for the current correlator in $k^{2}$ similarly to $[33,34,36,37] .{ }^{1}$ Such an

\footnotetext{
${ }^{1}$ We thank K. Tanaka for pointing this out.
}

analysis may lead to a more precise evaluation of the quarkonium matrix element and help to constrain the value of $\beta$. We leave this to future work. (ii) Our argument is similar in spirit to the vector meson dominance (VMD) hypothesis. Note that this is different from the VMD assumption used in many literature works on $J / \psi$ photoproduction mentioned in the introduction $[3,4,22,23]$. In these works, VMD has been applied to the incoming massless photon $\gamma \rightarrow J / \psi$. In photoproduction, this results in a significant mismatch between the initial and final virtualities $0 \rightarrow M^{2}$. Here, in a sense, we apply VMD in a reverse way to the outgoing $J / \psi \rightarrow \gamma^{*}$ (cf. [8,36,38]). While the difference in virtualities partly remains, this has little impact on the overall kinematics of the reaction because $\left|k^{2}-M^{2}\right| \ll Q^{2},|t|$. (iii) On the other hand, our approach is different from the nonrelativistic (NR)QCD framework [39] which is commonly used for quarkonium 
production in hadronic collisions. In NRQCD, the charm and anticharm quarks in the perturbative amplitude which couple to an external $J / \psi$ are both on-shell to leading order in the velocity expansion. However, in (12), $c$ and $\bar{c}$ are far off-shell with virtuality of the order of $Q^{2}$ (see below). Moreover, (9) assumes that $J / \psi$ is produced only in a color-singlet state. This is reasonable because near the threshold, all the energy has to be used to create a $J / \psi$, and there is little phase space for extra gluon emissions.

Let us now discuss the OPE. The current correlator on the right-hand side of (12) can be written as

$$
\begin{aligned}
& i \int d^{4} x d^{4} y e^{i k \cdot x-i q \cdot y}\left\langle p^{\prime}\left|\mathrm{T}\left\{\bar{c} \gamma^{\mu} c(x) J_{e m}^{\nu}(y)\right\}\right| p\right\rangle \\
& \quad \approx e_{f}(2 \pi)^{4} \delta^{(4)}\left(k+p^{\prime}-q-p\right) i \int d^{4} r e^{i r \cdot \frac{k+q}{2}}\left\langle p^{\prime}\left|\mathrm{T}\left\{\bar{c} \gamma^{\mu} c(r / 2) \bar{c} \gamma^{\nu} c(-r / 2)\right\}\right| p\right\rangle,
\end{aligned}
$$

where $r=x-y$. The product of currents can be expanded if the relative distance $\left|r^{\mu}\right|$ is small, which is the case when the momentum $\frac{k+q}{2}$ is deeply spacelike. From (6), near the threshold,

$$
t=(k-q)^{2}=M^{2}+q^{2}-2 k \cdot q \approx-\frac{m_{N}\left(M^{2}+Q^{2}\right)}{m_{N}+M} .
$$

Therefore,

$$
(k+q)^{2}=M^{2}+q^{2}+2 k \cdot q \approx 2 M^{2}-2 Q^{2}+\frac{m_{N}\left(M^{2}+Q^{2}\right)}{m_{N}+M} .
$$

This can be made arbitrarily negative by choosing $Q^{2} \gg M^{2}$. We need to also make sure that the large momentum $Q$ does not "leak" into the proton vertex which in practice means $Q^{2} \gg|t|$. Very close to the threshold, this is satisfied if $M \gg m_{N}$. As one goes away (but not too far away) from the threshold, the condition $Q^{2} \gg|t|$ is well satisfied when $t \sim t_{\min }{ }^{2}$ As we shall see in Sec. $\mathrm{V}, t \sim t_{\min }$ is the most interesting region.

However, for technical reasons the "symmetric" form (13) is not very convenient. Being a nonforward matrix element, it can be expressed in several different "frames"

$$
\begin{aligned}
i \int d^{4} r e^{i r \cdot \frac{k+q}{2}}\left\langle p^{\prime}\left|\mathrm{T}\left\{\bar{c} \gamma^{\mu} c\left(\frac{r}{2}\right) \bar{c} \gamma^{\nu} c\left(-\frac{r}{2}\right)\right\}\right| p\right\rangle & =i \int d^{4} r e^{i r \cdot q}\left\langle p^{\prime}\left|\mathrm{T}\left\{\bar{c} \gamma^{\mu} c(0) \bar{c} \gamma^{\nu} c(-r)\right\}\right| p\right\rangle \\
& =i \int d^{4} r e^{i r \cdot k}\left\langle p^{\prime}\left|\mathrm{T}\left\{\bar{c} \gamma^{\mu} c(r) \bar{c} \gamma^{\nu} c(0)\right\}\right| p\right\rangle .
\end{aligned}
$$

The meaning of the OPE is different in different frames. The final result must be the same, but this equivalence is often difficult to see. We shall return to this issue later. For the moment we find it most convenient to start with the middle expression of (16). We evaluate it as

$$
\begin{aligned}
i \int d^{4} r e^{i r \cdot q} \bar{c} \gamma^{\mu} c(0) \bar{c} \gamma^{\nu} c(-r)= & i \int d^{4} r e^{i r \cdot q}\left(\bar{c}(0) \gamma^{\mu} S(0,-r) \gamma^{\nu} c(-r)+\bar{c}(-r) \gamma^{\nu} S(-r, 0) \gamma^{\mu} c(0)\right) \\
& -i \int d^{4} r e^{i r \cdot q} \operatorname{Tr}\left[\gamma^{\mu} S(0,-r) \gamma^{\nu} S(-r, 0)\right]+\cdots
\end{aligned}
$$

where $S$ is the charm quark propagator in the presence of background gluon fields. Since we work in the regime $Q^{2} \gg M^{2}$, $\left|r^{\mu}\right|$ is typically much smaller than $1 / M$ and the heavy quark mass $m_{c} \approx M / 2$ can be neglected to first approximation. An important point of our approach is that we shall expand (17) in terms of local operators [40,41], instead of nonlocal lightcone correlators as is usually done in high energy scattering. Near the threshold, the role of light-cone directions appears to

\footnotetext{
${ }^{2}$ For example, if we set $W=4.4 \mathrm{GeV}$ and $Q^{2}=100 \mathrm{GeV}^{2}$, we find $\left|t_{\max }\right| \approx 52 \mathrm{GeV}^{2}$ and $\left|t_{\min }\right| \approx 10 \mathrm{GeV}^{2}$.
} 
be less conspicuous as the scattering is not necessarily in the forward direction. More importantly, the OPE with local operators is well suited for our purpose of establishing a connection to the D-term which is the matrix element of the (local) energy momentum tensor operator.

Consider the first line on the right-hand side of (17). The lowest contribution comes from the operator $\bar{c} \gamma^{\mu} \gamma_{5} c$, followed by higher dimensional operators such as $\bar{c} \gamma^{\mu} D^{\nu} c, \bar{c} \gamma^{\mu} F^{\alpha \beta} c$ and $\bar{c} \tilde{F}^{\alpha \beta} \gamma_{\beta} \gamma_{5} c$. The (nonforward) matrix elements of these operators measure the intrinsic charm contents of the proton which are in general believed to be tiny (see however, [5]). In this paper we simply neglect all of them, although they can be straightforwardly restored if need arises (see however, footnote 5 in Sec. V).

We thus focus on the second line of (17). Basically, we only keep dimension- 4 purely gluonic operators. This in particular includes the gluon part of the QCD energy momentum tensor

$$
T_{g}^{\alpha \beta}=-F^{\alpha \rho} F_{\rho}^{\beta}+\frac{g^{\alpha \beta}}{4} F^{\mu \nu} F_{\mu \nu},
$$

whose proton matrix element $\left\langle p^{\prime}\left|T_{g}^{\alpha \beta}\right| p\right\rangle$ is what we are ultimately interested in. However, certain higher dimension operators are a priori not suppressed. As in usual DIS or DVCS, the contribution of the leading twist operator with Lorentz spin- $j$ is proportional to $\left(2 q \cdot p / Q^{2}\right)^{j} \sim\left(1 / x_{B}\right)^{j}$ and $x_{B} \sim 1$ for our problem. The difficulty to sum over these higher spin operators with $j>2$ is the reason why the local version of the OPE is not commonly used in DVCS. Here, however, we do not attempt to perform this summation. Among the twist-two operators, the energy momentum tensor $T_{\alpha \beta}^{g}$ with $j=2$ dominates in the sum when $Q^{2}$ is sufficiently large. The contributions from the other twist-2 operators with spin $j>2$ are relatively suppressed because their anomalous dimensions are nonvanishing. Admittedly, the rate of this suppression is slow, only logarithmic in $Q^{2}$, so a large leverage in $Q^{2}$ is needed to isolate the spin-2 component. While this may seem a difficult task, we point out that a very similar problem exists in the current strategy to extract the quark D-term from the DVCS data [28-30]. The subtraction constant in the dispersion relation between the real and imaginary parts of the Compton form factor, commonly denoted by $\Delta(t)$ [27], is given by the sum of infinitely many Gegenbauer coefficients $\Delta\left(t, Q^{2}\right)=$ $d_{1}\left(t, Q^{2}\right)+d_{3}\left(t, Q^{2}\right)+\cdots$. In order to isolate the quark D-term $\propto d_{1}(t)$ which has the same anomalous dimension as the energy momentum tensor, one needs a large leverage in $Q^{2}$ to disentangle different moments. Assuming that such an analysis is feasible at the future EIC, we expect that the same can be done for the gluon D-term.

We shall work in Fock-Schwinger gauge $r_{\mu} A^{\mu}(r)=0$ for actual calculations. In this gauge, in the small- $r$ limit, the massless quark propagator in the background gluon field is given by, in $d=4-2 \varepsilon$ dimensions (see for example, $[42,43])$

$$
\begin{aligned}
S(r, 0)= & \frac{i \Gamma(d / 2)}{2 \pi^{d / 2}} \frac{\not t}{\left(-r^{2}\right)^{d / 2}}-\frac{i g \Gamma(1-\varepsilon)}{2^{5} \pi^{d / 2}} \frac{r_{\alpha} F_{\mu \nu}(0)}{\left(-r^{2}\right)^{1-\varepsilon}}\left(\gamma^{\alpha} \sigma^{\mu \nu}+\sigma^{\mu \nu} \gamma^{\alpha}\right)+i \frac{g^{2} \Gamma(-\varepsilon)\left(-r^{2}\right)^{\varepsilon}}{2^{6} \pi^{d / 2} N_{c}} r^{\alpha} F_{\alpha \rho}^{a} F_{a \beta}^{\rho}(0) \gamma^{\beta} \\
& -i \frac{g^{2} \Gamma(-\varepsilon)\left(-r^{2}\right)^{\varepsilon}}{3 \cdot 2^{6} \pi^{d / 2}}\left(\gamma^{\alpha} F_{\alpha \rho} F^{\rho}{ }_{\beta}(0) r^{\beta}+r^{\alpha} F_{\alpha \rho} F^{\rho}{ }_{\beta}(0) \gamma^{\beta}-\not F_{\alpha \beta} F^{\alpha \beta}(0)+\frac{2 \varepsilon \gamma^{\prime}}{r^{2}} r^{\alpha} F_{\alpha \rho} F^{\rho}{ }_{\beta}(0) r^{\beta}\right)+\cdots,
\end{aligned}
$$

where $F^{\alpha \beta}=F_{a}^{\alpha \beta} t^{a}$ with $\operatorname{Tr}\left(t^{a} t^{b}\right)=\delta^{a b} / 2$ and our convention for the covariant derivative is $D^{\mu}=\partial^{\mu}+i g A^{\mu}$. In the denominators, $r^{2}$ is short for $r^{2}-i \epsilon$. In (19), we have kept only the terms which contribute to dimension-4 gluonic operators $F F$. At first sight, the dimension-3 operators of the form $D_{\alpha} F_{\beta \gamma}$ are irrelevant because they are matrices in color space so when inserted in (17), they either vanish after tracing over color indices or lead to operators with dimension-5 or larger. However, for the present problem, it turns out that they cannot be neglected.
We shall discuss this later. Note that, since the FockSchwinger gauge breaks translational invariance, in general $S(r, 0) \neq S(0,-r)$. However, for the terms listed in (19), the relation $S(r, 0)=S(0,-r)$ actually holds.

In the second line of (17), the unit operator can be neglected because we are computing the nonforward amplitude $\left\langle p^{\prime}|1| p\right\rangle=0$. Consider then the $\mathcal{O}\left(g^{2} F F\right)$ terms in (19) which lead to a logarithmically enhanced contribution as implied by the prefactor $\Gamma(-\varepsilon)$. Taking the trace of the $g^{2} F F$ terms in (19) in color space, we find

$$
\operatorname{Tr}_{\text {color }}[S(r, 0)] \sim i \frac{g^{2} \Gamma(-\varepsilon)\left(-r^{2}\right)^{\epsilon}}{3 \cdot 2^{5} \pi^{d / 2}}\left(r^{\alpha} \gamma^{\beta} \hat{T}_{\alpha \beta}^{g}(0)-\frac{\varepsilon}{2 r^{2}} \gamma \gamma^{\prime}{ }_{\alpha \beta}^{g} r^{\alpha} r^{\beta}\right)
$$


where

$$
\hat{T}_{g}^{\alpha \beta} \equiv-F_{a}^{\alpha \rho} F_{a \rho}^{\beta}+\frac{g^{\alpha \beta}}{d} F_{a}^{\mu \nu} F_{\mu \nu}^{a},
$$

is the traceless part of the gluon part of the QCD energy momentum tensor. (20) explicitly shows that the logarithmic part is insensitive to the trace anomaly. Inserting the first term of (20) into the second line of (17), we find

$$
\begin{aligned}
- & i g^{2} \frac{\Gamma(-\varepsilon) \Gamma(d / 2)}{3 \cdot 2^{5} \pi^{d / 2+2}} \int d^{d} r e^{i r \cdot q} \frac{r^{\alpha} r_{\lambda}}{\left(-r^{2}+i \epsilon\right)^{d / 2-\varepsilon}} \operatorname{Tr}\left[\gamma^{\mu} \gamma^{\lambda} \gamma^{\nu} \gamma^{\beta}\right] \hat{T}_{\alpha \beta}^{g}(0) \\
& =\frac{\alpha_{s}}{12 \pi}\left(\frac{1}{\varepsilon}-\ln \left(-q^{2} / \mu^{2}\right)+1+\cdots\right)\left(\frac{g_{\lambda}^{\alpha}}{q^{2}}-2(1+\varepsilon) \frac{q^{\alpha} q_{\lambda}}{\left(q^{2}\right)^{2}}\right) \operatorname{Tr}\left[\gamma^{\mu} \gamma^{\lambda} \gamma^{\nu} \gamma^{\beta}\right] \hat{T}_{\alpha \beta}^{g}(0) \\
& \rightarrow-\frac{\alpha_{s}}{3 \pi}\left(\ln \left(-q^{2} / \mu^{2}\right)-1\right)\left(\frac{g_{\lambda}^{\alpha}}{q^{2}}-2 \frac{q^{\alpha} q_{\lambda}}{\left(q^{2}\right)^{2}}\right)\left(g^{\mu \lambda} g^{\nu \beta}-g^{\mu \nu} g^{\lambda \beta}+g^{\mu \beta} g^{\lambda \nu}\right) \hat{T}_{\alpha \beta}^{g}(0)-\frac{\alpha_{s}}{6 \pi} \frac{q^{\alpha} q_{\lambda}}{\left(\ell^{2}\right)^{2}} \operatorname{Tr}\left[\gamma^{\mu} \gamma^{\lambda} \gamma^{\nu} \gamma^{\beta}\right] \hat{T}_{\alpha \beta}^{g}(0) .
\end{aligned}
$$

Note that in the last step we have dropped the divergent piece $1 / \varepsilon$. It can be absorbed into the renormalization of the twist-two operator $\hat{T}_{c}^{\mu \nu} \sim \bar{c} \gamma^{(\mu} D^{\nu)} c$ contained in the first line of (17). The coefficient $\frac{\alpha_{s}}{3 \pi}$ can be identified with the anomalous dimension $\gamma_{c \leftarrow g}$ of this operator. As we already mentioned, we neglect the matrix element of (renormalized) $\hat{T}_{c}^{\mu \nu}$ so in practice the $1 / \varepsilon$ simply disappear.

The nonlogarithmic terms in (22) combine with those from the second term of (20) and the square of the $\mathcal{O}(g F)$ term in (19). After a tedious but straightforward calculation, we arrive at the total $\alpha_{s} F F$ contribution

$$
\begin{aligned}
\mathcal{A}^{\mu \nu} \equiv & i \int d^{4} r e^{i r \cdot q} \bar{c} \gamma^{\mu} c(0) \bar{c} \gamma^{\nu} c(-r) \\
\approx & -\frac{\alpha_{s}\left(\mu_{R}\right)}{3 \pi q^{2}}\left[2 \ln \left(-q^{2} / \mu_{R}^{2}\right)\left\{\left(g^{\mu \alpha}-\frac{q^{\mu} q^{\alpha}}{q^{2}}\right)\left(g^{\nu \beta}-\frac{q^{\nu} q^{\beta}}{q^{2}}\right)+\frac{q^{\alpha} q^{\beta}}{q^{2}}\left(g^{\mu \nu}-\frac{q^{\mu} q^{\nu}}{q^{2}}\right)\right\} \hat{T}_{\alpha \beta}^{g}(0)\right. \\
& \left.-2 \frac{q^{\alpha} q^{\beta}}{q^{2}}\left(g^{\mu \nu}-\frac{q^{\mu} q^{\nu}}{q^{2}}\right) \hat{T}_{\alpha \beta}^{g}(0)+3 \frac{q_{\alpha} q_{\beta}}{q^{2}} F^{\mu \alpha} F^{\nu \beta}(0)\right],
\end{aligned}
$$

where the operators are defined at the scale $\mu_{R}$. This is manifestly transverse with respect to $q$, i.e., $q_{\mu} \mathcal{A}^{\mu \nu}=$ $\mathcal{A}^{\mu \nu} q_{\nu}=0$, as a consequence of the Ward-Takahashi (WT) identity. In Appendix A, we show that the forward matrix element of (23) reproduces the 1-loop coefficient functions of the DIS structure functions. However, (23) has an obvious problem. The tensor $\mathcal{A}^{\mu \nu}$ is transverse with respect to $q^{\mu}$ and $q^{\nu}$, but this is because we have started with the middle expression in (13). In the present problem, gauge invariance rather implies $k^{\mu} \mathcal{A}_{\mu \nu}=\mathcal{A}_{\mu \nu} q^{\nu}=0$. Actually, problems of this kind typically arise in offforward kinematics. It is known that ensuring the electromagnetic gauge invariance of DVCS amplitudes is a highly nontrivial issue $[44,45]$. The leading order (leading twist) result does not fully satisfy the WT identity, and one has to include higher twist corrections to restore it. In the context of OPE, this amounts to including operators with total derivatives [46]. In Appendix B, we demonstrate that the dimension-3 operators $D_{\alpha} F_{\beta \gamma}$ which were neglected in (17) indeed give rise to total derivative operators. This calculation suggests that a complete treatment of the problem requires the inclusion of dimension-5 and even dimension6 operators in the expansion (17), which is beyond the scope of this work. Here instead, we suggest an ad hoc solution of the problem. In (23), we set $q^{2}=-\mu_{R}^{2}$ to eliminate the logarithmic terms. In the remainder terms, we implement the following minimal modifications ${ }^{3}$ to make $A^{\mu \nu}$ transverse with respect to $k^{\mu}$ and $q^{\nu}$, and symmetric in $q$ and $k$

$$
\begin{aligned}
\mathcal{A}^{\mu \nu} & \rightarrow-\frac{\alpha_{s}}{3 \pi(q \cdot k)^{2}}\left[-2 q^{\alpha} k^{\beta}\left(g^{\mu \nu}-\frac{q^{\mu} k^{\nu}}{q \cdot k}\right) \hat{T}_{\alpha \beta}^{g}\right. \\
& \left.+3 k_{\alpha} q_{\beta} F^{\mu \alpha} F^{\nu \beta}\right],
\end{aligned}
$$

\footnotetext{
${ }^{3}$ There is an ambiguity when replacing $q^{2}$ with $q \cdot k=$ $q^{2}-q \cdot \Delta$, since $q^{2} \rightarrow\left(\frac{q+k}{2}\right)^{2}=q^{2}-q \cdot \Delta+\Delta^{2} / 4$ seems to be an equally good choice [cf. (13)]. However, the difference is subleading because $q^{2} \approx 2 q \cdot k \gg \Delta^{2}$ in the present kinematics, see (14). This ambiguity can only be resolved by including the dimension-6 operator $\partial^{2} T_{\alpha \beta}$.
} 
where the coupling and the operators are evaluated at the scale $\mathcal{O}\left(Q^{2}\right)$. In the "leading-twist" approximation, one can further simplify [see (A2)]

$-F^{\mu \alpha} F^{\nu \beta} \approx \frac{1}{2}\left(g^{\mu \nu} \hat{T}_{g}^{\alpha \beta}-g^{\mu \beta} \hat{T}_{g}^{\alpha \nu}-g^{\alpha \nu} \hat{T}_{g}^{\mu \beta}+g^{\alpha \beta} \hat{T}_{g}^{\mu \nu}\right)$.

Actually, since we are neglecting the twist- 2 operators with spin $j>2$, it is not entirely consistent to include anything beyond (25) as it corresponds to twist-4 effects. Still, for phenomenological purpose it may be interesting to include at least the trace part of $T_{g}^{\alpha \beta}$ in order to assess the impact of the trace anomaly.

\section{TWO-GLUON FORM FACTORS}

In order to compute the actual cross section, we need to parametrize the nonforward matrix element of two-gluon operators in (24) in terms of form factors. First we have the gravitational form factors at our disposal [47]

$$
\left\langle p^{\prime}\left|T_{g}^{\mu \nu}\right| p\right\rangle=\bar{u}\left(p^{\prime}\right)\left[A_{g} \gamma^{(\mu} P^{\nu)}+B_{g} \frac{P^{(\mu} i \sigma^{\nu) \alpha} \Delta_{\alpha}}{2 m_{N}}+D_{g} \frac{\Delta^{\mu} \Delta^{\nu}-g^{\mu \nu} \Delta^{2}}{4 m_{N}}+\bar{C}_{g} m_{N} g^{\mu \nu}\right] u(p),
$$

where $\Delta^{\mu}=p^{\prime \mu}-p^{\mu}, P^{\mu} \equiv \frac{p^{\mu}+p^{\mu}}{2}$ and $A^{(\mu} B^{\nu)} \equiv\left(A^{\mu} B^{\nu}+A^{\nu} B^{\mu}\right) / 2$. All four form factors are functions of $t=\Delta^{2}$ and the renormalization scale $\mu_{R}$ in the $\overline{\mathrm{MS}}$ scheme. $D_{g}$ is the gluon part of the D-term form factor which we are mainly interested in. (In the literature often the notation $C_{g}=D_{g} / 4$ is often used.) The $\bar{C}_{g}$ form factor is related to the trace anomaly [25]. The traceless part reads

$$
\left\langle p^{\prime}\left|\hat{T}_{g}^{\mu \nu}\right| p\right\rangle=\bar{u}\left(p^{\prime}\right)\left[A_{g} \gamma^{(\mu} P^{\nu)}+B_{g} \frac{P^{(\mu} i \sigma^{\nu) \alpha} \Delta_{\alpha}}{2 m_{N}}+\frac{D_{g}}{4 m_{N}}\left(\Delta^{\mu} \Delta^{\nu}-\frac{g^{\mu \nu}}{d} \Delta^{2}\right)-\frac{m_{N} g^{\mu \nu}}{d}\left(A_{g}+\frac{\Delta^{2}}{4 m_{N}^{2}} B_{g}\right)\right] u(p) .
$$

Next consider the two gluon operator with four open indices

$$
\left\langle p^{\prime}\left|-F_{a}^{\mu \alpha} F_{a}^{\nu \beta}\right| p\right\rangle \text {. }
$$

Its most general parametrization consistent with parity, Hermiticity and time-reversal symmetry is ${ }^{4}$

$$
\begin{aligned}
\left\langle p^{\prime}\left|-F_{a}^{\mu \alpha} F_{a}^{\nu \beta}\right| p\right\rangle= & \frac{A}{2} \bar{u}\left(p^{\prime}\right)\left(g^{\mu \nu} \gamma^{(\alpha} P^{\beta)}-g^{\mu \beta} \gamma^{(\alpha} P^{\nu)}-g^{\alpha \nu} \gamma^{(\mu} P^{\beta)}+g^{\alpha \beta} \gamma^{(\mu} P^{\nu)}\right) u(p) \\
& +\frac{B}{4 m_{N}} \bar{u}\left(p^{\prime}\right)\left(g^{\mu \nu} i \sigma^{(\alpha \lambda} \Delta_{\lambda} P^{\beta)}-g^{\mu \beta} i \sigma^{(\alpha \lambda} \Delta_{\lambda} P^{\nu)}-g^{\alpha \nu} i \sigma^{(\mu \lambda} \Delta_{\lambda} P^{\beta)}+g^{\alpha \beta} i \sigma^{(\mu \lambda} \Delta_{\lambda} P^{\nu)}\right) u(p) \\
& +\frac{D}{8 m_{N}} \bar{u}\left(p^{\prime}\right)\left(g^{\mu \nu} \Delta^{\alpha} \Delta^{\beta}-g^{\alpha \nu} \Delta^{\mu} \Delta^{\beta}+g^{\alpha \beta} \Delta^{\mu} \Delta^{\nu}-g^{\mu \beta} \Delta^{\alpha} \Delta^{\nu}\right) u(p) \\
& +\frac{W}{3} m_{N} \bar{u}\left(p^{\prime}\right)\left(g^{\mu \nu} g^{\alpha \beta}-g^{\mu \beta} g^{\alpha \nu}\right) u(p) \\
& +\frac{X}{2 m_{N}^{2}} \bar{u}\left(p^{\prime}\right)\left(\left(\gamma^{\mu} \Delta^{\alpha}-\gamma^{\alpha} \Delta^{\mu}\right)\left(P^{\nu} \Delta^{\beta}-P^{\beta} \Delta^{\nu}\right)+\left(P^{\mu} \Delta^{\alpha}-P^{\alpha} \Delta^{\mu}\right)\left(\gamma^{\nu} \Delta^{\beta}-\gamma^{\beta} \Delta^{\nu}\right)\right) u(p) \\
& +\frac{Y}{m_{N}^{3}} \bar{u}\left(p^{\prime}\right)\left(P^{\mu} \Delta^{\alpha}-P^{\alpha} \Delta^{\mu}\right)\left(P^{\nu} \Delta^{\beta}-P^{\beta} \Delta^{\nu}\right) u(p) \\
& +\frac{Z}{4 m_{N}} \bar{u}\left(p^{\prime}\right)\left(i \sigma^{\mu \alpha}\left(P^{\nu} \Delta^{\beta}-P^{\beta} \Delta^{\nu}\right)+i \sigma^{\nu \beta}\left(P^{\mu} \Delta^{\alpha}-P^{\alpha} \Delta^{\mu}\right)\right) u(p) .
\end{aligned}
$$

The seven form factors can be partly constrained by requiring consistency with (26). Contracting the indices $\alpha \beta$ in (30), we get

\footnotetext{
${ }^{4}$ Terms which contain the antisymmetric tensor $\epsilon^{\mu \alpha \rho \lambda}$ are not independent. For example, the following identity holds

$$
i \bar{u}^{\prime} \epsilon^{\mu \alpha \rho \lambda} \gamma_{5} \gamma_{\rho} \bar{P}_{\lambda} u=m_{N} \bar{u}^{\prime} i \sigma^{\mu \alpha} u+\frac{1}{2} \bar{u}^{\prime}\left(\Delta^{\mu} \gamma^{\alpha}-\Delta^{\alpha} \gamma^{\mu}\right) u .
$$
}




$$
\begin{aligned}
\left\langle p^{\prime}\left|-F_{a}^{\mu \alpha} F_{a \alpha}^{\nu}\right| p\right\rangle= & \frac{A}{2} \bar{u}\left(p^{\prime}\right)\left(m_{N} g^{\mu \nu}+2 \gamma^{(\mu} P^{\nu)}\right) u(p)+\frac{B}{4 m_{N}} \bar{u}\left(p^{\prime}\right)\left(g^{\mu \nu} i \sigma^{\alpha \lambda} \Delta_{\lambda} P_{\alpha}+2 i \sigma^{(\mu \lambda} \Delta_{\lambda} P^{\nu)}\right) u(p) \\
& +\frac{D}{8 m_{N}} \bar{u}\left(p^{\prime}\right)\left(g^{\mu \nu} \Delta^{2}+2 \Delta^{\mu} \Delta^{\nu}\right) u(p)+W m_{N} g^{\mu \nu} \bar{u}\left(p^{\prime}\right) u(p) \\
& +\frac{X}{m_{N}^{2}} \bar{u}\left(p^{\prime}\right)\left(P^{(\mu} \gamma^{\nu)} \Delta^{2}+m_{N} \Delta^{\mu} \Delta^{\nu}\right) u(p)+\frac{Y}{m_{N}^{3}} \bar{u}\left(p^{\prime}\right)\left(P^{\mu} P^{\nu} \Delta^{2}+P^{2} \Delta^{\mu} \Delta^{\nu}\right) u(p) \\
& +\frac{Z}{4 m_{N}} \bar{u}\left(p^{\prime}\right)\left(2 i \sigma^{(\mu \alpha} P^{\nu)} \Delta_{\alpha}+\Delta^{\mu} \Delta^{\nu}\right) u(p) \\
= & A_{g} \bar{u}\left(p^{\prime}\right) \gamma^{(\mu} P^{\nu)} u(p)+\left(\frac{A}{2}+W+\frac{2 D_{g}+D}{8 m_{N}^{2}} \Delta^{2}+\frac{B \Delta^{2}}{8 m_{N}^{2}}\right) g^{\mu \nu} m_{N} \bar{u}\left(p^{\prime}\right) u(p) \\
& +\frac{B_{g}}{2 m_{N}} \bar{u}\left(p^{\prime}\right) i \sigma^{(\mu \lambda} \Delta_{\lambda} P^{\nu)} u(p)+\frac{D_{g}}{4 m_{N}} \bar{u}\left(p^{\prime}\right)\left(\Delta^{\mu} \Delta^{\nu}-g^{\mu \nu} \Delta^{2}\right) u(p),
\end{aligned}
$$

where in the second equality we used the following relations which can easily be obtained by term-by-term comparison:

$$
\begin{gathered}
A+\frac{\Delta^{2}}{m_{N}^{2}}(X+Y)=A_{g}, \\
B+Z-\frac{\Delta^{2}}{m_{N}^{2}} Y=B_{g}, \\
D+4 X+4 Y+\left(Z-\frac{\Delta^{2}}{m_{N}^{2}} Y\right)=D_{g} .
\end{gathered}
$$

We see that only two linear combinations of $X, Y, Z$ enter these relations.

By comparing the coefficients of $g^{\mu \nu}$, one should be able to obtain another relation between $W$ and $\bar{C}_{g}$. However, this is nontrivial due to the presence of the QCD trace anomaly.
If one naively contracts the indices $\mu \nu$ in (31) and computes the matrix element of $T_{g}^{\mu \nu}$ by forming the linear combination (18), one ends up with a wrong relation $\bar{C}_{g}=-A_{g} / 4$ (in the forward limit) and $W$ is undetermined. The problem is intimately tied to operator renormalization. In dimensional regularization, the following innocent-looking relation does not hold

$$
g_{\mu \nu}\left(F^{\mu \alpha} F_{\alpha}^{\nu}\right) \neq F^{2}
$$

Namely, operator renormalization and trace operation do not commute. The correct way to proceed is to write

$$
-F_{a}^{\mu \alpha} F_{a \alpha}^{\nu}=T_{g}^{\mu \nu}-\frac{g^{\mu \nu}}{4} F^{2}
$$

on the left-hand side of (31) and sum over the indices $\mu \nu$ using (26) and (31). This gives

$$
\left\langle p^{\prime}\left|F^{2}\right| p\right\rangle=\left[-2 A_{g}-4 W+4 \bar{C}_{g}-\left(\frac{3 D_{g}}{2}+\frac{B_{g}}{2}-4(X+Y)-Z+\frac{\Delta^{2}}{m_{N}^{2}} Y\right) \frac{\Delta^{2}}{m_{N}^{2}}\right] m_{N} \bar{u}\left(p^{\prime}\right) u(p)
$$

On the other hand, the matrix element $\left\langle p^{\prime}\left|F^{2}\right| p\right\rangle$ has to be carefully evaluated in a chosen regularization scheme [25,26] (see also [48]). In dimensional regularization, it is given by a linear combination of the gravitational form factors, see Eq. (13) of Ref. [13]

$$
\left\langle p^{\prime}\left|F^{2}\right| p\right\rangle=\left[K_{g}\left(A_{g}+4 \bar{C}_{g}\right)+K_{q}\left(A_{q}+4 \bar{C}_{q}\right)+\left(K_{g} B_{g}+K_{q} B_{q}-3 K_{g} D_{g}-3 K_{q} D_{q}\right) \frac{\Delta^{2}}{4 m_{N}^{2}}\right] m_{N} \bar{u}\left(p^{\prime}\right) u(p)
$$

where the quark gravitational form factors $A_{q}, B_{q}$, $D_{q}, \bar{C}_{q}$ are defined analogously to (26) for the quark part of the energy momentum tensor. The coefficients $K_{q, g}$ are defined in [13] and can be evaluated, in principle, to arbitrary order in perturbation theory. At the moment, the three-loop results are available $[25,26]$.
They depend on the number of flavors and the renormalization scale via the QCD coupling $\alpha_{s}\left(\mu_{R}\right)$. (37) and (38) give a complicated relation between $X, Y$, $Z, W$ and the quark and gluon gravitational form factors. In the forward limit $t=0$ it somewhat simplifies and we find 


$$
4 W(0)=4 \bar{C}_{g}(0)\left(1-K_{g}+K_{q}\right)-\left(2+K_{g}-K_{q}\right) A_{g}(0)-K_{q},
$$

where we used $A_{q}(0)+A_{g}(0)=1$ and $\bar{C}_{q}(t)+\bar{C}_{g}(t)=0$. The relation to the parameter $b$ often used in the literature [49] is

$$
b \equiv \frac{\left\langle p\left|\left(1+\gamma_{m}\right) \sum m_{f} \bar{q}_{f} q_{f}\right| p\right\rangle}{2 m_{N}^{2}}, \quad 1-b=\frac{\left\langle p\left|\frac{\beta(g)}{2 g} F^{2}\right| p\right\rangle}{2 m_{N}^{2}}=\frac{\beta}{2 g}\left(\left(A_{g}(0)+4 \bar{C}_{g}(0)\right)\left(K_{g}-K_{q}\right)+K_{q}\right),
$$

where $\gamma_{m}$ is the mass anomalous dimension. $b$ is the partition of the trace anomaly into the quark and gluon condensates. It is scheme and scale dependent.

\section{NUMERICAL RESULTS}

In this section we show numerical results for the differential cross section based on the formula (24). We do not intend to perform a complete calculation which is anyway not possible at the moment as it requires the detailed knowledge of all seven form factors $A(t), B(t), \ldots$. On the other hand, some information about the gravitational form factors $A_{g}, B_{g}, D_{g}, \bar{C}_{g}$ is already available in the literature. Based on this, we consider two interesting cases which allow us to make a quantitative prediction. Case 1: We use the "leading-twist" approximation (25) and keep only the traceless part of the energy momentum tensor (27). As explained in Sec. III, in doing so we assume that the contributions from the twist- 2 operators with spin $j>2$ can be either neglected or separated out by using a large leverage in $Q^{2}$. Case 2: We evaluate the full two-gluon operators (24) including the trace part of the energy momentum tensor. While this is not a consistent approximation (because we keep the twist-4 effect and neglect the twist-2, spin- $j>2$ contributions), it is an instructive exercise to assess the impact of the trace anomaly. In both cases, we set $B_{g}=0$ following the suggestion from lattice QCD (see, e.g., [50]) that this form factor is numerically small. In Case 2, we also set $X=$ $Y=Z=0$ as we know nothing about these form factors. On the other hand, the $W$ form factor is related to the trace anomaly and will be given full consideration.

We use the following parametrization of the gravitational form factors

$$
\begin{aligned}
A_{g}(t) & =\frac{A_{g}(0)}{\left(1-t / m_{A}^{2}\right)^{2}}, & A_{q}(t) & =\frac{1-A_{g}(0)}{\left(1-t / m_{A}^{2}\right)^{2}}, \\
D_{g}(t) & =\frac{D_{g}(0)}{\left(1-t / m_{C}^{2}\right)^{3}}, & \bar{C}_{g}(t) & =\frac{\bar{C}_{g}(0)}{\left(1-t / m_{A}^{2}\right)^{2}} .
\end{aligned}
$$

The tripole form of the D-term is motivated by the quark counting rule [51]. Since the form factors are evaluated at a large scale $\mu_{R}^{2}=Q^{2}$, to first approximation we can use the asymptotic results

$$
A_{q}(0) \approx \frac{n_{f}}{4 C_{F}+n_{f}}, \quad A_{g}(0) \approx \frac{4 C_{F}}{4 C_{F}+n_{f}}, \quad D_{q}(0) \approx \frac{n_{f}}{4 C_{F}} D_{g}(0)
$$

with $C_{F}=\frac{N_{c}^{2}-1}{2 N_{c}}$ and $n_{f}=3$ represents the number of light flavors in the proton. ${ }^{5}$ The value $D_{g}(0)$ is our main interest and should be determined by future experiments. Here, for the sake of demonstration, we use the results of a recent lattice simulation $D_{g}(0)=-7.2 \quad\left(C_{g}(0)=-1.8\right)$ with $m_{A}=1.13 \mathrm{GeV}$ and $m_{C}=0.76 \mathrm{GeV}$ at $\mu_{R}=2 \mathrm{GeV}$ [53]. (We neglect the scale dependence of these parameters.) On the other hand $\bar{C}_{g}$ at zero momentum transfer is related to the QCD trace anomaly [25]. Asymptotically $\mu_{R}^{2} \rightarrow \infty$,

\footnotetext{
${ }^{5}$ As we commented in Sec. III, we neglect the charm quark operators in the expansion (17). Let us nevertheless give a rough estimate of its impact. The charm quark contribution to the proton momentum is $A_{c} \approx 0.02$ in the relevant $Q^{2}$ range (see for example [52]). Comparing this to $\alpha_{s} A_{g}=0.64 \alpha_{s}$, we see that the correction due to intrinsic charm is less than $20 \%$. Still, this may have to be included in more precise calculations in future.
}

$$
\bar{C}_{g}(0) \approx \frac{1}{4}\left(\frac{n_{f}}{4 C_{F}+n_{f}}+\frac{2 n_{f}}{3 \beta_{0}}\right)-\frac{1}{4}\left(\frac{2 n_{f}}{3 \beta_{0}}+1\right) \frac{b}{1+\gamma_{m}}
$$

where $b$ is introduced in (40). To one-loop, $\beta_{0}=11 N_{c} / 3-$ $2 n_{f} / 3$ and $\gamma_{m}=\frac{3 C_{F} \alpha_{s}}{2 \pi}$. A more precise expression can be found in $[25,26]$.

Under these assumptions, (37) and (38) reduce to a simple formula

$$
\begin{aligned}
4 W(t)= & 4 \bar{C}_{g}(t)\left(1-K_{g}+K_{q}\right)-\left(2+K_{g}+\frac{1-A_{g}(0)}{A_{g}(0)} K_{q}\right) A_{g}(t) \\
& +3 D_{g}(t)\left(K_{g}+\frac{n_{f}}{4 C_{F}} K_{q}-2\right) \frac{\Delta^{2}}{4 m_{N}^{2}}
\end{aligned}
$$

For simplicity, we use the one-loop result $K_{q, g}$ 


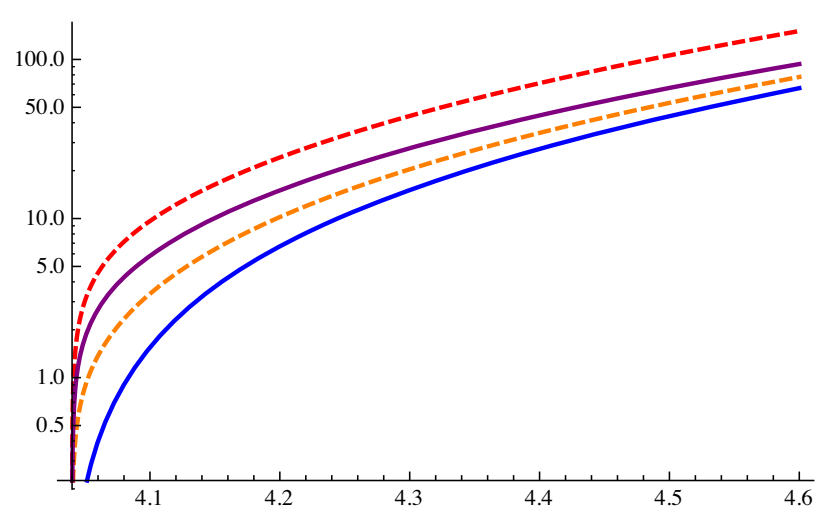

(a) Total cross section (in fb) as a function of $W$ (in $\mathrm{GeV}$ ).

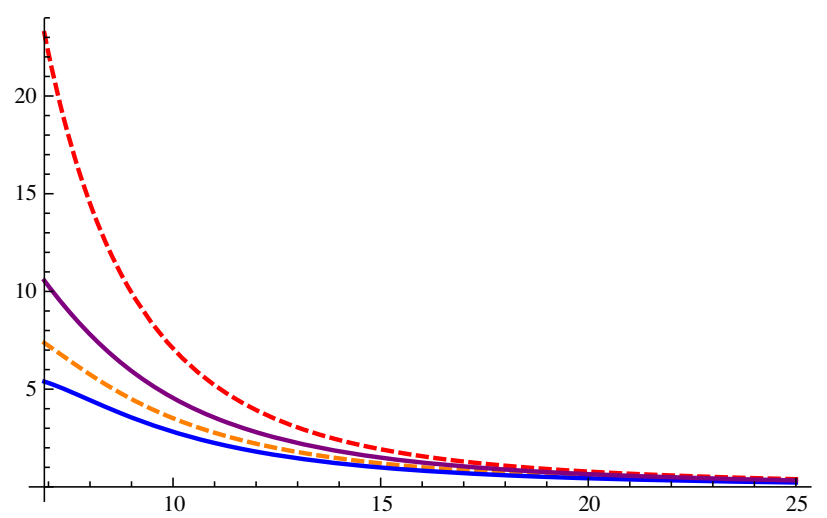

(b) Differential cross section (in $\mathrm{fb} / \mathrm{GeV}^{2}$ ) at $W=4.4 \mathrm{GeV}$ as a function of $|t|$ (in $\mathrm{GeV}^{2}$ ).

FIG. 1. $J / \psi$ total and differential cross sections at $Q^{2}=64 \mathrm{GeV}^{2}$. The upper and lower dashed curves correspond to Case 1 with $D_{g}=0$ and $D_{g}=-7.2$, respectively. The upper and lower solid curves correspond to Case $2, D_{g}=-7.2$, with $b=1$ and $b=0$, respectively.

$$
K_{g}=\left(\mathcal{C}_{g F}-\frac{\mathcal{C}_{g m}}{\mathcal{C}_{q m}} \mathcal{C}_{q F}\right)^{-1}, \quad K_{q}=-\frac{\mathcal{C}_{g m}}{\mathcal{C}_{q m}} K_{g}
$$

where $\left[\alpha_{s}=\alpha_{s}\left(Q^{2}\right)\right]$

$$
\begin{aligned}
& \mathcal{C}_{q m}=1+\frac{C_{F}}{3 \pi} \alpha_{s}, \quad \mathcal{C}_{q F}=\frac{n_{f}}{12 \pi} \alpha_{s}, \\
& \mathcal{C}_{g m}=\frac{7 C_{F}}{6 \pi} \alpha_{s}, \quad \mathcal{C}_{g F}=-\frac{11 N_{c}}{24 \pi} \alpha_{s} .
\end{aligned}
$$

See [26] for the three-loop result. Finally, the square of the prefactor in (9) is evaluated as [including the factor $e_{f}$ from (13)]

$$
\frac{e_{c}^{4} e^{2} M^{4}}{g_{\gamma J / \psi}^{2}} \approx 24.6,
$$

where we set $e_{c}=2 / 3, M=3.1 \mathrm{GeV}, \Gamma_{e^{+} e^{-}}=5.55 \mathrm{keV}$ and $\alpha_{e m}=e^{2} / 4 \pi=1 / 137$. The corresponding value for $\Upsilon$ is

$$
\frac{e_{b}^{4} e^{2} M^{4}}{g_{\gamma \Upsilon}^{2}} \approx 19.4,
$$

where $e_{b}=-1 / 3, M=9.46 \mathrm{GeV}$ and $\Gamma_{e^{+} e^{-}}=1.34 \mathrm{keV}$. The parameter $\beta$ should be determined by fitting the data (for example the total cross section at some value of $W$ ) for each quarkonium species. In the numerical results below we set $|\beta|=1$.

In Fig. 1, we show the total and differential cross sections for $J / \psi$ at $Q=8 \mathrm{GeV}, \alpha_{s}(Q)=0.2$. The latter is evaluated at $W=4.4 \mathrm{GeV}$. In both plots, the upper and lower dashed curves correspond to Case 1 with $D_{g}=0$ and $D_{g}=-7.2$, respectively. We see a dramatic impact of the gluon D-term. ${ }^{6}$ A negative (positive) D-term tends to shrink (enhance) the differential cross section. The same tendency has been observed in [9] in the case of photoproduction $Q^{2}=0$. The upper and lower solid curves correspond to Case 2 with $b=1$ (zero gluon condensate) and $b=0$ (zero quark condensate), respectively. We see that the dependence on the parameter $b$ is significant. We also see that the gluon condensate tends to reduce the cross section, which is actually opposite to what was found in [9]. It is not clear to us whether this is due to the fact that different processes were considered (photoproduction vs leptoproduction), or perhaps due to the deficiency of the model used in [9].

Next, in Fig. 2 we show the result for $\Upsilon$ at $Q=18 \mathrm{GeV}$, $\alpha_{s}(Q)=0.16$. Near the threshold $\left(W_{t h}=10.4 \mathrm{GeV}\right)$, the cross section becomes very small. In the right panel we selected a somewhat large value $W=12.5 \mathrm{GeV}$ considering the realistic luminosity of EIC. Again we see a large effect of the D-term. However, the impact of the trace anomaly and the split between $b=1$ and $b=0$ are barely visible. This suggests that, at least theoretically, $\Upsilon$ production is better suited for the purpose of extracting the D-term.

In photoproduction, the $J / \psi$ total cross section is about $1 \mathrm{nb}$ at $W=4.5 \mathrm{GeV}$ [16]. In leptoproduction, we see that the cross section is several orders of magnitude smaller. For $\Upsilon$ production, there is another 2 orders of magnitude suppression. Besides, what is computed here is the cross section in the $\gamma^{*} p$ subsystem. Thus, near-threshold leptoproduction is a luminosity-hungry observable. Moreover, as explained in Sec. III, one needs a large leverage in $Q^{2}$ to extract the D-term. Given these requirements, we think that

\footnotetext{
${ }^{6}$ Remember that we neglected the RG evolution of $D_{g}$ from $\mu_{R}=2 \mathrm{GeV}$ to $Q=8 \mathrm{GeV}$. The value $\left|D_{g}(0)\right|$ at the scale $Q$ will be smaller than 7.2 so the actual difference between the two dashed curves is expected to be smaller.
} 


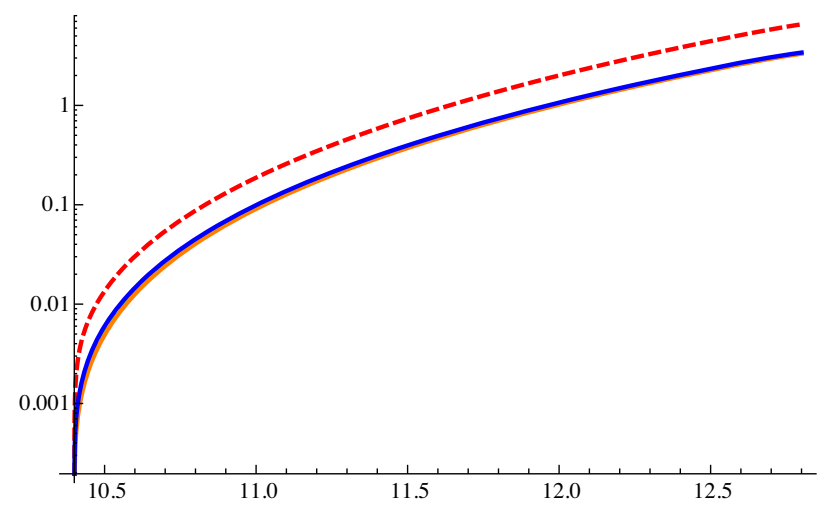

(a) Total cross section (in $\mathrm{fb}$ ) as a function of $W$ (in $\mathrm{GeV}$ ).

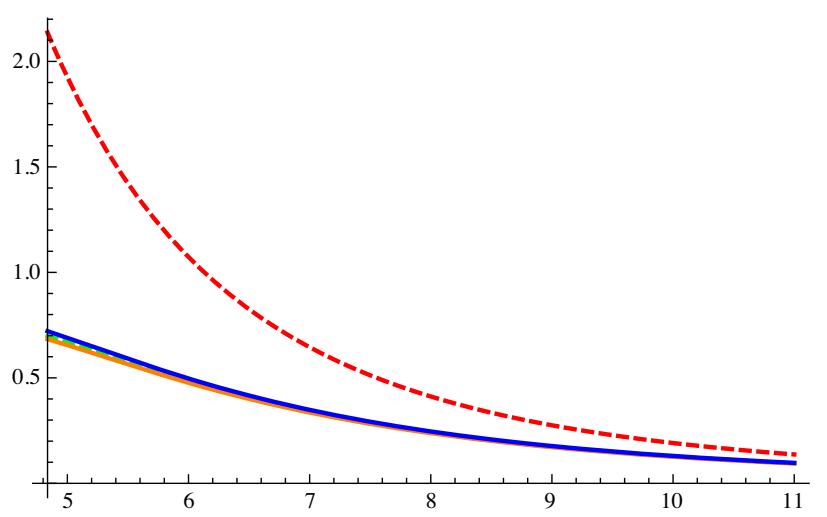

(b) Differential cross section (in $\mathrm{fb} / \mathrm{GeV}^{2}$ ) at $W=12.5 \mathrm{GeV}$ as a function of $|t|\left(\right.$ in $\left.\mathrm{GeV}^{2}\right)$.

FIG. 2. $\Upsilon$ total and differential cross sections at $Q^{2}=18^{2} \mathrm{GeV}^{2}$. See the caption of Fig. 1 for the explanation of each curve.

the best place to test our proposal is $J / \psi$ (and possibly also $\Upsilon$ ) production in the high luminosity mode of EIC.

\section{CONCLUSION}

In this paper we have proposed a novel strategy to compute the cross section of near-threshold quarkonium production at large momentum transfer. Compared to photoproduction, near-threshold leptoproduction has so far attracted much less attention due to the lack of strong phenomenological motivations. We have demonstrated that the process is useful for probing the gluon D-term, quite complementary to the ongoing effort to extract the quark D-term in DVCS. The possible impact of the D-term on the differential cross section $d \sigma / d t$ has been already pointed out in the case of photoproduction using holography $[9,14]$. In leptoproduction at large $Q^{2} \gg M^{2}$, the problem can be studied within the perturbative framework. Moreover, at the subleading level the cross section is also sensitive to the value of the parameter $b$ defined in Eq. (40) which characterizes the structure of the QCD trace anomaly. The proposed measurements require high luminosity and a large leverage in $Q^{2}$. The only machine that can deliver these requirements is the future EIC.

Our analysis in this paper is only the first step and there are a number of directions for future research. In particular, it is interesting to see if a similar approach can be applied to photoproduction using the heavy quark mass as a hard scale. On the phenomenological side, the contribution from the Bethe-Heitler process $e^{-} \rightarrow e^{-} \gamma^{*}$ needs to be investigated as $J / \psi$ and $\Upsilon$ are reconstructed from lepton pairs in actual experiments, and this should be implemented in realistic simulations to study experimental feasibility and detector requirements at the EIC. The seven form factors introduced in (30) can be calculated in lattice QCD. The values of $t$ considered in this paper are rather large, and the extrapolation to the forward limit is a serious challenge. Lattice calculations of these form factors will be very valuable in this respect.

\section{ACKNOWLEDGMENTS}

We are grateful to Jian-Wei Qiu and Kazuhiro Tanaka for discussions and critical comments, and Peter Schweitzer for correspondence. This work is supported by the U.S. Department of Energy, Office of Science, Office of Nuclear Physics, under contract No. DE- SC0012704, and in part by Laboratory Directed Research and Development (LDRD) funds from Brookhaven Science Associates and by Grant No. 2019/33/B/ST2/02588 of the National Science Center in Poland.

\section{APPENDIX A: DIS COEFFICIENT FUNCTIONS}

As a consistency check, let us compute the forward matrix element of (23) in a single proton state and keep only the twist- 2 contribution. In this approximation, we can write

$$
\left\langle p\left|\hat{T}_{g}^{\alpha \beta}\right| p\right\rangle=2 A_{g} p^{\alpha} p^{\beta},
$$

where $A_{g}$ is the fraction of the proton momentum carried by gluons. We then decompose the operator $F^{\mu \alpha} F^{\nu \beta}$ as ${ }^{7}$

$$
\begin{aligned}
F^{\mu \alpha} F^{\nu \beta}= & \frac{1}{d-2}\left(g^{\mu \nu} F^{\lambda \alpha} F_{\lambda}^{\beta}-g^{\mu \beta} F^{\lambda \alpha} F_{\lambda}{ }^{\nu}\right. \\
& \left.-g^{\alpha \nu} F^{\lambda \mu} F_{\lambda}^{\beta}+g^{\alpha \beta} F^{\lambda \mu} F_{\lambda}{ }^{\nu}\right) \\
& +\frac{1}{(d-2)(d-1)}\left(g^{\mu \beta} g^{\nu \alpha}-g^{\mu \nu} g^{\alpha \beta}\right) g_{\rho \sigma} F^{\rho \lambda} F_{\lambda}^{\sigma} \\
& +C^{\mu \alpha \nu \beta},
\end{aligned}
$$

\footnotetext{
${ }^{7}$ This decomposition is mathematically identical to that of the Riemann tensor in general relativity. The trace part $T_{\alpha \beta}$ is an analog of the Ricci tensor which represents the matter content and $C^{\mu \alpha \nu \beta}$ is an analog of the Weyl tensor which represents the gravity degrees of freedom.
} 
and extract the energy momentum tensor component $T_{g}^{\alpha \beta} \sim-F^{\alpha \lambda} F_{\lambda}^{\beta}$. The remainder tensor $C^{\mu \alpha \nu \beta}$ has the same symmetry as $F^{\mu \alpha} F^{\nu \beta}$ except that it is traceless with respect to any pair of indices. Its forward matrix element vanishes. We thus have

$$
\left\langle p\left|-F^{\mu \alpha} F^{\nu \beta}\right| p\right\rangle \approx A_{g}\left(g^{\mu \nu} p^{\alpha} p^{\beta}-g^{\mu \beta} p^{\alpha} p^{\nu}-g^{\alpha \nu} p^{\mu} p^{\beta}+g^{\alpha \beta} p^{\mu} p^{\nu}\right) .
$$

This gives

$$
\begin{aligned}
& i \int d^{4} r e^{i r \cdot q}\left\langle p\left|\bar{c} \gamma^{\mu} c(0) \bar{c} \gamma^{\nu} c(-r)\right| p\right\rangle \\
& \quad \approx 2 A_{g} \frac{\alpha_{s}}{\pi\left(q^{2}\right)^{2}}\left[\frac{2}{3}(p \cdot q)^{2}\left(g^{\mu \nu}-\frac{q^{\mu} q^{\nu}}{q^{2}}\right)-\left(\frac{2}{3} \ln \frac{-q^{2}}{\mu_{R}^{2}}-\frac{1}{2}\right) q_{\alpha} q_{\beta}\left(g^{\mu \nu} p^{\alpha} p^{\beta}-g^{\mu \beta} p^{\alpha} p^{\nu}-g^{\nu \alpha} p^{\mu} p^{\beta}+g^{\alpha \beta} p^{\mu} p^{\nu}\right)\right] .
\end{aligned}
$$

From this one can read off the known one-loop coefficient functions for the DIS structure functions

$$
C_{2,2}^{G}=\left(\frac{2}{3} \ln \frac{-q^{2}}{\mu_{R}^{2}}-\frac{1}{2}\right) \frac{\alpha_{s}}{4 \pi}, \quad C_{L, 2}^{G}=\frac{2}{3} \frac{\alpha_{s}}{4 \pi},
$$

in the notation of [54].

\section{APPENDIX B: TOTAL DERIVATIVE OPERATORS}

In this appendix, we give an example of how operators with total derivatives enter the calculation. We return to (19) and include dimension-3 operators $D_{\alpha} F_{\beta \gamma}$ which have been previously neglected

$$
\begin{gathered}
S(r, 0) \sim \frac{4 g}{3 \cdot 2^{6} \pi^{d / 2}}\left(-r^{2}\right)^{\varepsilon}\left[\Gamma(-\varepsilon) D^{\rho} F_{\rho \lambda} \gamma^{\lambda}+\frac{1}{r^{2}}\left(\not r^{\lambda} D^{\rho} F_{\rho \lambda}+r^{\alpha} r^{\beta} D_{\beta} F_{\alpha \lambda} \gamma^{\lambda}+3 i r^{\alpha} r^{\beta} D_{\alpha} \tilde{F}_{\beta \lambda} \gamma^{\lambda} \gamma_{5}\right)\right], \\
S(0,-r) \sim \frac{4 g}{3 \cdot 2^{6} \pi^{d / 2}}\left(-r^{2}\right)^{\varepsilon}\left[\Gamma(-\varepsilon) D^{\rho} F_{\rho \lambda} \gamma^{\lambda}+\frac{1}{r^{2}}\left(\not r^{\lambda} D^{\rho} F_{\rho \lambda}+r^{\alpha} r^{\beta} D_{\beta} F_{\alpha \lambda} \gamma^{\lambda}-3 i r^{\alpha} r^{\beta} D_{\alpha} \tilde{F}_{\beta \lambda} \gamma^{\lambda} \gamma_{5}\right)\right] .
\end{gathered}
$$

This can be derived following $[42,43]$. Note that the last term proportional to $\gamma_{5}$ breaks the naive relation $S(r, 0)=S(0,-r)$. Let us focus only on the singular term $\propto \Gamma(-\varepsilon)$ which is sufficient to demonstrate our point. Its contribution to the current correlator is

$$
\begin{aligned}
& \frac{\alpha_{s} \Gamma[1-\varepsilon]}{3 \cdot 2^{5} \pi^{d-1}} \int d^{d} r e^{-i r \cdot q} \frac{r_{\alpha}}{\left(-r^{2}\right)^{1-2 \varepsilon}} \Gamma(-\varepsilon) \tilde{F}^{\alpha \lambda} D^{\sigma} F_{\sigma \rho} \operatorname{Tr}\left[\gamma^{\mu} \gamma_{\lambda} \gamma_{5} \gamma^{\nu} \gamma^{\rho}\right] \\
& =i \frac{\alpha_{s}(4 \pi)^{\varepsilon}}{3 \pi} \frac{\Gamma(2+\varepsilon) \Gamma[1-\varepsilon]}{\Gamma(1-2 \varepsilon)} \frac{q_{\alpha}\left(-q^{2}\right)^{-\varepsilon}}{\left(q^{2}\right)^{2}} \epsilon^{\mu \lambda \nu \rho} \tilde{F}_{\lambda}^{\alpha} D^{\sigma} F_{\sigma \rho} \Gamma(-\varepsilon) \\
& =-i \frac{\alpha_{s}}{3 \pi\left(\ell^{2}\right)^{2}}\left(\frac{1}{\varepsilon}+1-\ln \frac{\left(-q^{2}\right)}{\mu_{R}^{2}}\right)\left(q^{\mu} \partial_{\rho} T_{g}^{\rho \nu}-q^{\nu} \partial_{\rho} T_{g}^{\rho \mu}-F^{\mu \nu} q^{\rho} D^{\sigma} F_{\sigma \rho}\right),
\end{aligned}
$$

where we used the identity $\partial_{\nu} T_{g}^{\mu \nu}=F_{\nu}^{\mu} D_{\alpha} F^{\alpha \nu}$. Note that this is antisymmetric in $\mu$ and $\nu$. The $1 / \varepsilon$ divergence in (B3) is absorbed into the renormalization of the operator

$$
\bar{c} \gamma^{\mu} g \tilde{F}_{\lambda}^{\alpha} \gamma_{\lambda} \gamma_{5} \gamma^{\nu} c-(\mu \leftrightarrow \nu) \sim \epsilon^{\mu \lambda \nu \rho} \bar{c} \gamma_{\rho} g \tilde{F}_{\lambda}^{\alpha} c
$$

which comes from the first line of (17). The remaining terms contain total derivative operators. In the nonforward matrix element, the derivative operator is replaced by the momentum transfer $\left\langle p^{\prime}\left|\partial_{\rho} T_{g}^{\rho \nu}\right| p\right\rangle=i \Delta_{\rho}\left\langle p^{\prime}\left|T_{g}^{\rho \nu}\right| p\right\rangle$ where
$\Delta_{\rho}=q_{\rho}-k_{\rho}$. This is how, in principle, total derivative operators from higher dimensional terms can restore the WT identity through the addition of $\Delta$ corrections. However, (B3) is not sufficient to make the logarithmic terms in (23) transverse with respect to $k^{\mu}$. For that, we would need operators like $\partial^{\nu} T_{g}^{\mu \beta}$ and $g^{\mu \nu} \partial_{\alpha} T_{g}^{\alpha \beta}$. We presume that the missing terms come from the dimension- 5 and dimension- 6 operators in the expansion of $S(r, 0)$. We leave this to a future work. 
[1] B. Gittelman, K. M. Hanson, D. Larson, E. Loh, A. Silverman, and G. Theodosiou, Phys. Rev. Lett. 35, 1616 (1975).

[2] U. Camerini, J. G. Learned, R. Prepost, C. M. Spencer, D. E. Wiser, W. W. Ash, R. L. Anderson, D. M. Ritson, D. J. Sherden, and C. K. Sinclair, Phys. Rev. Lett. 35, 483 (1975).

[3] T. H. Bauer, R. D. Spital, D. R. Yennie, and F. M. Pipkin, Rev. Mod. Phys. 50, 261 (1978); 51, 407(E) (1979).

[4] D. Kharzeev, H. Satz, A. Syamtomov, and G. Zinovjev, Eur. Phys. J. C 9, 459 (1999).

[5] S. J. Brodsky, E. Chudakov, P. Hoyer, and J. M. Laget, Phys. Lett. B 498, 23 (2001).

[6] L. Frankfurt and M. Strikman, Phys. Rev. D 66, 031502 (2002).

[7] P. Bosted et al., Phys. Rev. C 79, 015209 (2009).

[8] O. Gryniuk and M. Vanderhaeghen, Phys. Rev. D 94, 074001 (2016).

[9] Y. Hatta and D. L. Yang, Phys. Rev. D 98, 074003 (2018).

[10] J. Xu and F. Yuan, Phys. Lett. B 801, 135187 (2020).

[11] Y. Hatta, M. Strikman, J. Xu, and F. Yuan, Phys. Lett. B 803, 135321 (2020).

[12] I. Strakovsky, D. Epifanov, and L. Pentchev, Phys. Rev. C 101, 042201 (2020).

[13] Y. Hatta, A. Rajan, and D. L. Yang, Phys. Rev. D 100, 014032 (2019).

[14] K. A. Mamo and I. Zahed, Phys. Rev. D 101, 086003 (2020).

[15] R. Wang, X. Chen, and J. Evslin, arXiv:1912.12040.

[16] A. Ali et al. (GlueX Collaboration), Phys. Rev. Lett. 123, 072001 (2019).

[17] S. Joosten and Z. Meziani, Proc. Sci., QCDEV2017 (2018) 017 [arXiv:1802.02616].

[18] An Assessment of U.S.-Based Electron-Ion Collider Science, National Academies of Sciences, Engineering, and Medicine (The National Academies Press, Washington, DC, 2018).

[19] A. Accardi et al., Eur. Phys. J. A 52, 268 (2016).

[20] C. A. Aidala et al., arXiv:2002.12333.

[21] F. Yuan (private communication).

[22] J. Pumplin and W. Repko, Phys. Rev. D 12, 1376 (1975).

[23] V. D. Barger and R. J. N. Phillips, Phys. Lett. 58B, 433 (1975).

[24] M. E. Luke, A. V. Manohar, and M. J. Savage, Phys. Lett. B 288, 355 (1992).

[25] Y. Hatta, A. Rajan, and K. Tanaka, J. High Energy Phys. 12 (2018) 008.

[26] K. Tanaka, J. High Energy Phys. 01 (2019) 120.
[27] M. V. Polyakov and P. Schweitzer, Int. J. Mod. Phys. A 33, 1830025 (2018).

[28] V. D. Burkert, L. Elouadrhiri, and F. X. Girod, Nature (London) 557, 396 (2018).

[29] K. Kumeriki, Nature (London) 570, E1 (2019).

[30] H. Moutarde, P. Sznajder, and J. Wagner, Eur. Phys. J. C 79, 614 (2019).

[31] M. Lomnitz and S. Klein, Phys. Rev. C 99, 015203 (2019).

[32] J. C. Collins, L. Frankfurt, and M. Strikman, Phys. Rev. D 56, 2982 (1997).

[33] V. Braun, D. Ivanov, A. Lenz, and A. Peters, Phys. Rev. D 75, 014021 (2007).

[34] V. Braun, D. Ivanov, and A. Peters, Phys. Rev. D 77, 034016 (2008).

[35] E. R. Berger, M. Diehl, and B. Pire, Eur. Phys. J. C 23, 675 (2002).

[36] G. Altarelli and G. Preparata, Phys. Lett. B 39, 371 (1972).

[37] B. Pasquini, M. Gorchtein, D. Drechsel, A. Metz, and M. Vanderhaeghen, Eur. Phys. J. A 11, 185 (2001).

[38] M. F. Lutz and M. Soyeur, Nucl. Phys. A760, 85 (2005).

[39] G. T. Bodwin, E. Braaten, and G. P. Lepage, Phys. Rev. D 51, 1125 (1995); 55, 5853(E) (1997).

[40] K. Watanabe, Prog. Theor. Phys. 67, 1834 (1982).

[41] Z. Chen, Nucl. Phys. B525, 369 (1998).

[42] E. V. Shuryak and A. I. Vainshtein, Nucl. Phys. B201, 141 (1982).

[43] I. Balitsky and V. M. Braun, Nucl. Phys. B311, 541 (1989).

[44] I. V. Anikin, B. Pire, and O. V. Teryaev, Phys. Rev. D 62 , 071501 (2000).

[45] A. V. Belitsky and A. V. Radyushkin, Phys. Rep. 418, 1 (2005).

[46] V. M. Braun and A. N. Manashov, J. High Energy Phys. 01 (2012) 085.

[47] X. Ji, Phys. Rev. Lett. 78, 610 (1997).

[48] S. Rodini, A. Metz, and B. Pasquini, arXiv:2004.03704.

[49] X. D. Ji, Phys. Rev. Lett. 74, 1071 (1995).

[50] C. Alexandrou, M. Constantinou, K. Hadjiyiannakou, K. Jansen, C. Kallidonis, G. Koutsou, A. Vaquero Avils-Casco, and C. Wiese, Phys. Rev. Lett. 119, 142002 (2017).

[51] K. Tanaka, Phys. Rev. D 98, 034009 (2018).

[52] R. D. Ball, V. Bertone, M. Bonvini, S. Carrazza, S. Forte, A. Guffanti, N. P. Hartland, J. Rojo, and L. Rottoli (NNPDF Collaboration), Eur. Phys. J. C 76, 647 (2016).

[53] P. E. Shanahan and W. Detmold, Phys. Rev. Lett. 122, 072003 (2019).

[54] S. A. Larin, P. Nogueira, T. van Ritbergen, and J. A. M. Vermaseren, Nucl. Phys. B492, 338 (1997). 